\title{
ON THE DOMAIN OF TRACES AND SEQUENTIAL COMPOSITION
}

\author{
Marta Z. Kwiatkowska ${ }^{1}$ \\ Department of Computing Studies \\ University of Leicester \\ Leicester LE1 7RH \\ United Kingdom \\ e-mail (JANET): mzk@uk.ac.le
}

\begin{abstract}
An enhancement of Mazurkiewicz's trace theory with infinite traces is presented. Infinite traces have been obtained by introducing the trace preorder relation on possibly infinite strings. It is shown that the extension gives rise to the domain of traces in the sense of Scott and a complete metric space. Sequential composition (concatenation) of possibly infinite traces is also considered. The difficulty of finding an appropriate concatenation of infinite traces is a consequence of the concatenation of finite traces being non-uniformly continuous wrt the metric for traces. A natural extension of the concatenation operation for finite traces is proposed; the extended operation is total, yields a generalization of Levi's lemma for infinite traces, but is non-associative.
\end{abstract}

\section{INTRODUCTION}

Trace theory, originating from Mazurkiewicz [Maz77], has recently gained popularity as a semantic model of non-interleaving concurrency. Although the properties of finite traces are relatively well-known, see e.g. [Maz84, Aa88], it seems that infinite traces have not yet been fully investigated. This is undesirable as infinite behaviours are required in order to establish certain properties of reactive systems, for example liveness [Pnu86], and also has impact on fairness issues [Kwi89t, Kwi90t]. The corresponding enhancement of finite strings with infinite ones has been known for some time, see e.g. [BoN80]; it gives rise to an infinitary monoid, a domain in the sense of Scott and a complete metric trace. An interesting question arises as to what properties of the algebra of finite and infinite strings generalize onto the algebra of traces.

In this paper, we investigate the monoid and order-theoretic properties of the algebra of finite and infinite traces. First, trace equivalence over infinite strings and the notion of trace prefix ordering for possibly infinite traces are introduced. It is shown that this ordering gives rise to a Scott domain and a complete metric space. Then, the difficulty of finding an appropriate extension of the concatenation of finite traces to infinite ones is discussed, and a total operation of sequential composition is proposed. The operation allows to generalize Levi's lemma to possibly infinite traces, but unfortunately is nonassociative. Finally, possible ways of introducing an acceptable operation are discussed. This paper is a 
contribution to the search for an adequate semantic framework for non-interleaving concurrency, which uses topological theory originating from Nivat, see e.g. [BoN80].

An alternative approach has been independently developed in [Gas90]. While preparing the final version of this paper, it has come to our knowledge that Levi's lemma has also been extended to infinite traces in [Die91].

\section{PRELIMINARIES}

This section introduces notation conventions and recalls standard definitions, which can be found in e.g. [GHK80, BoN80, Law87].

Let $(P, \leq)$ be a poset. For $X \subseteq P$ and $x \in P$ write: $\downarrow X=\{y \in P \mid y \leq x$ for some $x \in X\}, \uparrow X=$ $\{\mathrm{y} \in \mathrm{P} \mid \mathrm{X} \leq \mathrm{y}$ for some $\mathrm{x} \in \mathrm{X}\} . \mathrm{X} \subseteq \mathrm{P}$ is a lower set (also prefix-closed) iff $\mathrm{X}=\downarrow \mathrm{X}$. $\mathrm{X} \subseteq \mathrm{P}$ is an upper set (or upward-closed) iff $\mathrm{X}=\uparrow \mathrm{X} . \mathrm{X} \subseteq \mathrm{P}$ is directed iff it is non-empty and every pair of elements $\mathrm{x}, \mathrm{y} \in \mathrm{X}$ have a bound $\mathrm{z}$ also in $\mathrm{X} . \mathrm{X} \subseteq \mathrm{P}$ is an ideal iff it is a directed lower set.

To distinguish between orderings, we shall use $\leq$ and $\sqsubset$; the least upper bounds and greatest lower bounds are denoted by $\amalg$ and $\Pi$ respectively.

Let $(\mathrm{D}, \mathrm{c})$ be a complete partial order (cpo) and $\mathrm{x}, \mathrm{y} \in \mathrm{D}$. We say $\mathrm{x}$ is essentially below (or way below) $\mathrm{y}$, denoted $\mathrm{x} \subseteq^{\text {fin }} \mathrm{y}$, iff given a directed set $\mathrm{M} \subseteq \mathrm{D}$ such that $\mathrm{y} \subseteq U \mathrm{M}$, then there exists $\mathrm{z} \in \mathrm{M}$ such that $x \subseteq z$. Intuitively, $x$ is essentially below $y$ if $x$ is some finite approximation of $y$. For $X \subseteq D$ and $x \in D$ write: $\downarrow^{\text {fin }} X=\left\{y \in D \mid y\left\llcorner^{\text {fin }} x\right.\right.$ for some $\left.x \in X\right\}, \uparrow^{\text {fin }} X=\left\{y \in D \mid x \stackrel{\text { fin }}{ }^{\text {fin }}\right.$ for some $\mathrm{X} \in \mathrm{X}\}$.

Let $(D, \subseteq)$ be a cpo. $D$ is consistently complete iff every subset $\mathrm{X} \subseteq \mathrm{D}$ bounded in $\mathrm{D}$ has a least upper bound. $\mathrm{x} \in \mathrm{D}$ is a finite element (also called compact) if, whenever $\mathrm{M} \subseteq \mathrm{D}$ is directed and $x \subseteq \sqcup M$, then there exists $y \in M$ such that $x \subseteq y$. Equivalently, $x$ is finite iff $x \subseteq^{\text {fin }} x$. The set of all finite elements of $D$ is denoted $B_{D}$. $D$ is algebraic iff, for every $x \in D$, the set $M=\left[y \in B_{D} \mid y \subseteq x\right]=$ $\downarrow^{\text {fin }} \mathrm{x}$ is directed and $\cup M=x . D$ is a Scott domain iff $D$ is algebraic, consistently complete and $B_{D}$ is countable.

Example 2.1. Consider the poset $P=\{0,1\}^{*}$ of all finite strings over $\{0,1\}$ (including the empty string $\varepsilon$ ) with the usual prefix order denoted by $\leq$. The ideal completion $\operatorname{Id}(P)$ can be identified with all finite and infinite strings. Id(P) is a Scott domain. $\{0,1)^{*}$ are finite elements. The essentially below relation $\leq^{\text {fin }}$ is the inherited order.

We use $A^{\omega}$ to represent the set of all infinite sequences over $A$, and $A^{\infty}$ is the union of $A^{*}$ and $A^{\omega}$. The prefix order over $A^{\infty}$ will be denoted by $\leq$ and is defined as follows:

$$
\forall x, y \in A^{\infty}: x \leq y \Leftrightarrow \forall i \in N-\{0\}:(i \leq l e n(x) \Rightarrow x(i)=y(i))
$$

where $x(i)$ denotes the ith symbol of the sequence $x$ if it exists, and $\varepsilon$ otherwise.

Concatenation is extended onto $A^{\infty}$ [BoN80] by taking as the concatenation of sequences $x, y$ :

$$
\begin{array}{ll}
\forall x \in A^{*}, y \in A^{\omega}: & \text { the infinite sequence } x y \\
\forall x \in A^{\omega}, y \in A^{\infty}: & \text { the infinite sequence } x .
\end{array}
$$

Concatenation of strings will be denoted as juxtaposition. $A^{\infty}$, together with the above concatenation, forms an infinitary monoid [BoN80]. This is a natural extension of the concatenation over finite strings. $A^{\infty}$ is a complete metric space wrt to the ultrametric: 


$$
d_{\text {str }}(x, y)= \begin{cases}2^{-k} & \text { if } \exists n \in N-\{0\}: x(n) \neq y(n) \\ 0 & \text { iff } \forall n \in N-\{0\} ; x(n)=y(n)\end{cases}
$$

where $k=\min \{n \in N-\{0\} \mid x(n) \neq y(n)\}$. The concatenation in $A^{*}$ is uniformly continuous wrt the metric $d_{\text {str }},\left(A^{*}, d_{\text {str }}\right)$ is dense in $\left(A^{\infty}, d_{\text {str }}\right)$, and hence the concatenation has a canonical continuous extension on $A^{\circ}$.

Applications of the topological theory of complete metric spaces in semantics originate from Nivat, see e.g. [BoN80]. An alternative approach is due to Scott. Given cpo's D, E, a function $\mathrm{f:D} \rightarrow \mathrm{E}$ is Scott-continuous iff $f$ is monotone, i.e. $x \leq y$ implies $f(x) \leq f(y)$, and $f(\sqcup M)=\sqcup f(M)$ for every directed set $M$. The concatenation on $A^{*}$ is not Scott-continuous in both arguments (it is not even monotone, consider e.g. $(a, b) \leq(a a, b b)$, but $a b \leq a a b b)$. However, the following simple modification to the definitions results in concatenation becoming Scott continuous. Let $A$ be a (finite) alphabet and $\sqrt{ } \notin A$ denote the termination symbol. Consider the set:

$$
A \sqrt{ }{ }^{\infty}=A^{*} \cup A^{*} \sqrt{ } \cup A^{\omega}
$$

of strings with the prefix ordering $\leq$ as usual. It is a Scott domain, and the concatenation ; defined by:

$$
x ; y= \begin{cases}x^{\prime} y & \text { if } x=x^{\prime} \sqrt{ } \\ x & \text { otherwise }\end{cases}
$$

is Scott continuous.

\section{INFINITE TRACES}

\section{Trace Equivalence and Finite Traces}

In this section we recall basic definitions concerning trace theory; for complete presentations see e.g. [Maz77, AaR88, Maz84, Maz89]. Trace languages constitute an abstraction of concurrent behaviour derivable from the notion of causal independency. Define the independency to be an irreflexive and symmetric relation $\mathrm{t} \subseteq \mathrm{A} \times \mathrm{A}$. Two actions are independent if they can happen concurrently without affecting the result, and dependent otherwise. A concurrent alphabet $(A, 1)$ is formed from a finite set of action symbols $A$ and an independency $l \subseteq A \times A$. Given a concurrent alphabet $(A, l)$ one may obtain trace equivalence $[\mathrm{Maz} 77]$ as the least congruence $\equiv$ fin in the monoid of finite strings $\left(A^{*}, ., \varepsilon\right)$ such that $a \mathrm{i} b \Rightarrow a b \equiv b a$. It then follows that for all $v, w \in A^{*}, v \equiv$ fin $w$ iff $w$ can be obtained from $v$ through a finite number of transformations involving only permutations of consecutive independent symbols.

The equivalence class of a string is called a trace. Every trace should be thought of as containing the set of all possible sequentializations of a given (non-sequential) execution; two sequentializations are the same up to permutations of consecutive independent action symbols. For example, if $A=\{a, b, c\}$ and $t$ $=\{(a, b),(b, a)\}$ then:

$$
\begin{aligned}
& {[a b]=\{a b, b a\}} \\
& {[a c]=\{a c\}} \\
& {[a c b]=\{a c b\} .}
\end{aligned}
$$


The monoid structure induces an order relation over finite traces. A trace $\sigma$ is a prefix of $\tau$, denoted

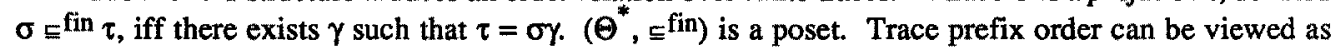
approximation ordering on executions, where each trace corresponds to a (global) state. [ $\varepsilon]$ is the initial state, incomparable prefices of the same trace represent states arising during concurrent execution, and $\tau_{1}$ ${ }_{\text {fin }} \tau_{2}$ means that $\tau_{1}$ is a partial execution leading to $\tau_{2}$. Fig. 1 shows a sample concurrent execution together with its prefices.

The following is a summary of properties of finite traces:

(i) $\left(\Theta^{*}, .,[\varepsilon]\right)$ is a cancellative monoid;

(ii) $\left(\Theta^{*}, \underline{\subseteq}^{\text {fin }}\right)$ is a consistently complete countable poset [Kwi89t].

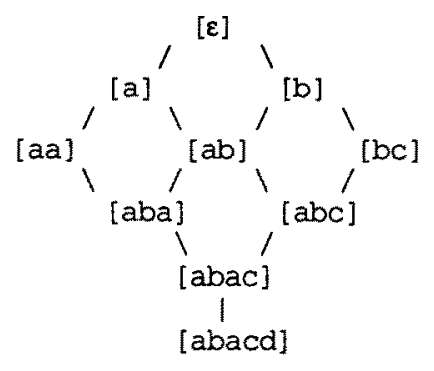

Fig. 1. The poset of all trace prefices of [abcad] with a $\mathbf{l} b$, a $\mathbf{l}$.

\section{Extending Trace Equivalence onto Infinite Strings}

We extend trace equivalence onto possibly infinite strings by introducing trace preorder $\leq_{\mathrm{tr}}$ for finite and infinite strings as follows:

$$
\mathrm{u} \leq_{\mathrm{tr}} \mathrm{v} \Leftrightarrow\left(\forall \mathrm{x} \leq \text { fin } \mathrm{u} \exists \mathrm{y} \leq \operatorname{fin}^{\mathrm{v}} \mathrm{v}\left(\exists \mathrm{z} \in \mathrm{A}^{*}: \mathrm{x} \leq \mathrm{z} \text { and } \mathrm{z} \equiv \text { fin } \mathrm{y}\right)\right)
$$

Observe that $\mathrm{u} \leq \mathrm{v}$ implies $\mathrm{u} \leq_{\mathrm{ur}} \mathrm{v}$, that is, the preorder $\leq_{\mathrm{x}}$ is weaker than the string prefix order $\leq$, in the sense that it ignores permutations of consecutive independent symbols. We now define trace equivalence in $A^{\infty}$, denoted $\equiv$, as the equivalence relation determined by the trace preorder $\leq_{\mathbb{t r}}$; more precisely, for all $x, y \in A^{\infty}$ :

$$
\mathrm{x} \equiv \mathrm{y} \Leftrightarrow\left(\mathrm{x} \leq_{\mathrm{rr}} \mathrm{y} \text { and } \mathrm{y} \leq_{\mathrm{rr}} \mathrm{x}\right)
$$

It can be shown that this definition coincides with trace equivalence $\equiv$ fin over $A^{*}$.

The set of all infinite traces over the concurrent alphabet $(A, 1)$ is denoted by $\Theta_{1}{ }^{\omega}$, and $\Theta_{1}{ }^{\infty}=$ $\Theta_{\mathfrak{l}}{ }^{*} \cup \Theta_{\mathfrak{l}}{ }^{\omega}$. The subscript $l$ will often be omitted.

Example 3.1. Consider $A=\{a, b, c\}$ with a $\imath$ b. It is easy to see that $a \leq_{t r} a b, a \leq_{t r} b a, a \leq_{t r} a c, a \unlhd_{t r}$ $c a, a b \leq_{t r} b a, b a \leq_{t r} a b, a \leq_{t r} a^{\omega}, a^{\omega} \leq_{t r}(a b)^{\omega}$, and $a^{\omega} \leq_{t r}(b a)^{\omega}$.

It should be emphasised that, unlike the definition of trace equivalence in the monoid of finite strings, our definition of $\equiv$ allows for an infinite number of permutations of two consecutive independent symbols. For example, $(a b)^{\omega} \equiv(b a)^{\omega}$ for $a \imath b$. 
An equivalent trace preorder definition has been independently introduced in [Gas90]. It is worth mentioning that there are other ways of defining trace equivalence, see e.g. [Shi85, Gas90], and other representations of traces, e.g. dependency graphs [AaR88] and vector languages [Shi85].

\section{Trace Prefix Ordering for Possibly Infinite Traces}

Trace prefix ordering on possibly infinite traces is determined by the preorder $\leq_{\mathrm{tr}}$. A (finite or infinite) trace $\sigma$ is a prefix of a (finite or infinite) trace $\tau$ over $C=(A, 1)$, denoted by $\sigma \subseteq \tau$, iff:

$$
\forall \mathrm{x} \in \sigma \exists \mathrm{y} \in \tau: \mathrm{x} \leq_{\mathrm{tx}} \mathrm{y} .
$$

The following property follows from the definition:

\section{Proposition 3.2.}

(i) If $\sigma \subseteq \tau$ then for all $\mathrm{y} \in \tau$ there exists $\mathrm{x} \in \sigma$ such that $\mathrm{x} \leq_{\mathrm{r}} \mathrm{y}$.

(ii) If $\sigma \equiv \tau$ then for all $\mathrm{x} \in \sigma, \mathrm{y} \in \tau: \mathrm{x} \leq_{\mathrm{tr}} \mathrm{y}$.

The restriction of the relation $\subseteq$ to the finite elements coincides with the ordering $\subseteq^{\text {fin }}$ over $\Theta^{*}$. The following may also be easily shown:

Proposition 3.3. Let $\sigma \in \Theta^{*}, \tau \in \Theta^{\infty}$ such that $\sigma \equiv \tau$. Then:

(i) For all $\mathrm{x} \in \sigma$ there exists $\mathrm{y} \in \tau$ such that $\mathrm{x} \leq \mathrm{y}$ (i.e. $\mathrm{x}$ is a string prefix of $\mathrm{y}$ ).

(ii) For all $\mathrm{y} \in \tau$ there exists $\mathrm{x} \in \sigma$ such that $\mathrm{x} \leq \mathrm{y}$.

Proposition 3.3(ii) does not extend onto infinite traces; for example $\left[a^{\omega}\right] \subseteq\left[b^{0}\right]$ when a $\mathrm{b}$, but for no $y \in\left[b a^{\omega}\right]=\left\{a^{*} b a^{\omega}\right\}$ do we have $a^{\omega} \leq y$.

The set of prefices of a given trace is directed, but not necessarily a total order like in the algebra of strings. As a consequence, in contrast to prefix ordering for strings, it is possible to show two distinct infinite traces, of which one dominates the other one. For example, the following holds for a $\mathrm{l} b$ :

$$
\left[(a)^{\omega}\right] \subseteq\left[b(a)^{\omega}\right] \subseteq\left[b b(a)^{\omega}\right] \subseteq \ldots \subseteq\left[(a b)^{\omega}\right] .
$$

If there exists a trace that dominates both $\tau_{1}$ and $\tau_{2}$ we say that $\tau_{1}$ and $\tau_{2}$ are consistent; otherwise they are inconsistent, in other words, two consistent traces correspond to consistent observations of a nonsequential behaviour, while inconsistent ones to observations that could not possibly have occurred during the same execution. If $\tau_{1} \equiv \tau_{2}$ or $\tau_{2} \equiv \tau_{1}$ we say that $\tau_{1}$ and $\tau_{2}$ are comparable; otherwise they are incomparable; two incomparable but consistent traces arise from concurrent execution. Unlike in the algebra of strings, two incomparable traces are not necessarily inconsistent.

A trace $\tau$ is maximal in $T \subseteq \Theta^{\infty}$ iff there does not exist a trace $\sigma \in \mathrm{T}$ such that $\tau \subseteq \sigma$ and $\sigma \neq \tau$. Maximal traces correspond to complete executions, whereas non-maximal traces are partial. The notion of maximality has impact on fairness issues [Kwi89t]. 


\section{PROPERTIES OF TRACES}

\section{The Domain of Traces}

In this section we investigate order-theoretic properties of $\left(\Theta^{\infty}, ㄷ\right)$. We show that $\left(\Theta^{\infty}, 5\right)$ is a generalization of $\left(A^{\infty}, \leq\right)$ in the sense that it forms a Scott domain which is isomorphic to $\left(A^{\infty}, \leq\right)$ in the case of $\imath=\varnothing$.

Lemma 4.1. Let $(\mathrm{P}, \leq)$ be a poset. Then:

(i) $\mathrm{X} \subseteq \mathrm{P}$ is directed $\Leftrightarrow \downarrow \mathrm{X}$ is directed.

(ii) If $\mathrm{X}$ is a finite directed subset of $\mathrm{P}$ then $\mathrm{LX}$ exists and belongs to $\mathrm{X}$.

\section{Proof.}

(i) Can be found in [GHK80].

(ii) By induction on cardinality of the set.

Theorem 4.2. Let $(\mathrm{A}, \mathrm{l})$ be a concurrent alphabet. Then $\left(\Theta^{\infty}, \subseteq\right)$ is a cpo.

\section{Froof.}

1) It is clear that $[\varepsilon]$ is the least element.

2) We prove that every directed subset $X$ of $\Theta^{\infty}$ has a least upper bound. By Lemma 4.1 it is sufficient to show the existence of least upper bounds of infinite directed sets $X \subseteq \Theta^{\infty}$ such that $X=\downarrow X$. Let $X \subseteq \Theta^{\infty}$ be such a set. Since $X \cap \Theta^{*}$ is countable, its elements can be ordered into a sequence $\left\{\sigma_{k} \mid k\right.$ $\in N$ ). We construct $\sqcup X$ as a limit of a monotonically increasing sequence in $X \cap \Theta^{*}$. Define the monotonically increasing family $M_{k}$ of directed subsets of $X \cap \Theta^{*}$ by:

$$
\begin{aligned}
& M_{0}=\varnothing \\
& M_{k}= \begin{cases}M_{k-1} \cup\left\{\sigma_{k}\right\} & \text { if } M_{k-1} \cup\left\{\sigma_{k}\right\} \text { is directed } \\
M_{k-1} \cup\left\{\sigma_{k}, \beta\right\} & \text { if } M_{k-1} \cup\left\{\sigma_{k}\right\} \text { is not directed and } \beta \text { is a bound for } M_{k-1} \cup\left\{\sigma_{k}\right\} .\end{cases}
\end{aligned}
$$

Note that $\beta$ always exists by the assumption of directedness of $X$. Also note that $X \cap \Theta^{*}=$ $U\left(M_{k} \mid k \in N\right\}$. Since each $M_{k}$ is finite and directed, $\sqcup M_{k}$ exists and belongs to $M_{k}$ by Lemma 4.1(ii). By construction of the family $M_{k}$ we have $M_{k} \subseteq M_{k+1}$, and hence:

$U M_{k}=U M_{k+1}$ for all $k \in N$.

Let $x_{k} \in U M_{k}, x_{k+1} \in U M_{k+1}$; then $x_{k} \leq_{t r} x_{k+1}$ by Proposition 3.2(ii). Since each $U M_{k} \in \Theta^{*}$ we have $x_{k} \in A^{*}$ for all $k \in N$ and by definition of trace preorder $s_{\mathrm{t}}$ :

$\exists z_{k+1}: x_{k} \leq z_{k+1}$ and $x_{k+1} \equiv$ fin $z_{k+1}$;

it follows that $z_{k+1} \in U \mathbf{M}_{k+1}$. We have thus shown:

$\forall \mathrm{k} \forall \mathrm{z}_{\mathrm{k}} \in U \mathrm{M}_{\mathrm{k}} \exists \mathrm{z}_{\mathrm{k}+1} \in U \mathrm{M}_{\mathrm{k}+1}: \mathrm{z}_{\mathrm{k}} \leq \mathrm{z}_{\mathrm{k}+1}$,

and hence it is possible to construct inductively a monotonic sequence $\left\{\mathrm{z}_{\mathrm{k}} \mid \mathrm{k} \in \mathrm{N}\right\} \subseteq \mathrm{A}^{*}$ starting from $\sqcup M_{0}$. Define $x=\sqcup\left[z_{k} \mid k \in N\right\}$ (exists because $A^{\infty}$ is a cpo), and take $\gamma=[x]_{1}$. It is easy to see that $\gamma$ is the least upper bound of $X$.

This concludes the proof. 
We now show $\left(\Theta^{\infty}, 5\right)$ is consistently complete. The following binary lefi cancellation operator, denoted $l: \Theta^{*} \times \Theta^{*} \rightarrow \Theta^{*}$, will be required:

$$
\forall \sigma, \gamma \in \Theta^{*}: \quad \sigma / \gamma=\tau \Leftrightarrow\left(\gamma \check{c}^{\text {fin }} \sigma \text { and } \gamma \tau=\sigma\right) \text {. }
$$

$\sigma / \gamma$ is pronounced $\sigma$ after $\gamma$, it denotes the continuation of $\sigma$ after its prefix $\gamma$ has been completed.

Lemma 4.3. Let $(\mathrm{P}, \leq)$ be a poset, and let $\mathrm{X} \subseteq \mathrm{P}$. Then the following conditions are equivalent:

(i) $\cup \mathrm{X}$ exists

(ii) $\amalg \downarrow \mathrm{X}$ exists.

And if these conditions are satisfied, then $\sqcup \mathrm{X}=\sqcup \downarrow \mathrm{X}$. Moreover, if every finite subset of $X$ has a least upper bound and if $\mathrm{F}$ denotes the set of all those finite least upper bounds, then $\mathrm{F}$ is directed and (i) and

(ii) are equivalent to:

(iii) UF exists

Under these circumstances, UX = UF.

Proof. Can be found in [GHK80].

Lemma 4.4. Let $(\mathrm{D}, \mathrm{5})$ be a cpo. Then the following are equivalent:

(i) (D, 5$)$ is consistently complete,

(ii) Every non-empty subset of D has a greatest lower bound.

\section{Proof.}

$\Rightarrow$ ) Observe $\Pi X$ equals $\sqcup L_{X}$ where $L_{X}$ denotes the set of lower bounds of $X$.

$\Leftrightarrow$ Observe $U X$ equals $\Pi U_{x}$ where $U_{x}$ denotes the set of upper bounds of $X$.

Lemma 4.5. Let $\sigma, \gamma \in \Theta^{*}, \beta \in \Theta^{\infty}$.

If $\sigma, \gamma \subseteq \beta$ then there exists $\delta \in \Theta^{*}$ such that $\delta=\sigma u \gamma$.

\section{Proof.}

Let $\sigma, \gamma \in \Theta^{*}, \beta \in \Theta^{\infty}$ such that $\sigma, \gamma \subseteq \beta$.

1) We show there exists $\beta^{\prime} \in \Theta^{*}$ such that $\sigma, \gamma \subseteq \beta^{\prime} \sqsubseteq \beta$. Let $x \in \sigma, y \in \gamma, z \in \beta$; then $x \leq_{t r} z$ and $\mathrm{y} \leq_{\mathrm{rr}} \mathrm{z}$ by Proposition 3.2. Since $\mathrm{x}$ and $\mathrm{y}$ are finite, we have by definition of $\leq_{\mathrm{tr}}$ that there exist finite prefices $z_{1}, z_{2}$ of $z$ such that $x \leq u_{1}$ and $y \leq u_{2}$ for some $u_{1} \equiv z_{1}, u_{2} \equiv z_{2}$. Let $\beta^{\prime}=\left[\max \left\{z_{1}, z_{2}\right\}\right]$. It is clear that $\sigma, \gamma \subseteq \beta^{\prime} \subseteq \beta$ and $\beta^{\prime} \in \Theta^{*}$.

2) We can now assume $\sigma, \gamma, \beta \in \Theta^{*}$ and $\sigma, \gamma \subseteq \beta$. We prove by induction on the length of $\sigma$ that $\delta=$ $\sigma u \gamma$ exists. If $\sigma=[\varepsilon]$ then take $\delta=\gamma$. It is clear that $\delta=\sigma\lrcorner \gamma$. Suppose $\sigma \neq[\varepsilon]$ and $[a] \subseteq \sigma$ for some $a \in A$. Since $\sigma \subseteq \beta$ we have $[a] \subseteq \beta$. There are two cases:

2a) $[\mathrm{a}] \subseteq \gamma$, and thus $\gamma=[\mathrm{a}](\gamma /[\mathrm{a}]) \subseteq \beta=[\mathrm{a}](\beta /[\mathrm{a}])$, which implies, by left cancellation law, that $\gamma /[a] \subseteq \beta /[a]$. By symmetry, $\sigma /[a] \subseteq \beta /[a]$. By induction on the length of $\sigma$ it follows that $\delta^{\prime}=$ $(\sigma /[\mathrm{a}]) \cup(\gamma /[\mathrm{a}])$ exists. It is easy to see that:

$$
\delta=\sigma u \gamma=[a] \delta^{\prime} \text {. }
$$

2b) [a] $\equiv \gamma$, from which it follows by a fairly straightforward argument (omitted, see [Kwi89t]) that $\gamma \subseteq$ $\beta /[a]$. By induction on the length of $\sigma$ we have $\delta^{\prime}=(\sigma /[a]) \cup \gamma$ exists. Finally, it is clear that:

$$
\delta=\sigma \cup \gamma=[a] \delta^{\prime}
$$

This concludes the proof. 


\section{Theorem 4.6.}

(i) $\quad\left(\Theta^{\infty}, \sqsubseteq\right)$ is consistently complete.

(ii) Every subset $\mathrm{X}$ of $\Theta^{\infty}$ has a greatest lower bound $\Pi \mathrm{X}$ wrt $ᄃ$.

\section{Proof.}

(i) Suppose $X \subseteq \theta^{\infty}$ bounded. We show $\cup X$ exists. Let $Y$ be a finite subset of $X$, then the existence of $\checkmark Y$ can be proved using induction and Lemma 4.5. Let $F$ denote the set of $U Y$ for all finite subsets $Y$ of $X$. Then $F$ is directed by Lemma 4.3 , and hence $U F$ exists because $\left(\Theta^{\infty}, 5\right)$ is a cpo (Theorem 4.2 ). Thus, since $\sqcup F=\sqcup X$ by Lemma 4.3 , we have shown that $\sqcup X$ exists.

(ii) Direct from Lemma 4.4 .

Finally, we prove that the set of all traces forms a domain in the sense of Scott.

Lemma 4.7. Let $(\mathrm{D}, 5)$ be a cpo. If $\mathrm{D}$ is consistently complete, then $\downarrow^{\mathrm{fin}} \mathrm{x}$ is directed for all $\mathrm{x} \in \mathrm{D}$.

Proof. See e.g. [Law87].

Theorem 4.8. Let $\mathrm{C}=(\mathrm{A}, \mathrm{l})$ be a concurrent alphabet. Then $\left(\Theta^{\infty}\right.$, 드 $)$ is a Scott domain.

Proof.

1) Since $\left(\Theta^{\infty}\right.$, ㄷ) is consistently complete (Proposition 4.6), it follows by Lemma 4.7 that $\downarrow^{\text {fin }} \sigma$ is directed for all $\sigma \in \Theta^{\infty}$, and the least upper bound of $\downarrow^{\text {fin }} \sigma$ exists. It is clear that $\sqcup \downarrow^{\text {in }} \sigma=\sigma$. Hence, $\left(\Theta^{\infty}, \sqsubseteq\right.$ ) $)$ is algebraic.

2) We now show that $\Theta^{*}$ are the finite elements. Suppose $\sigma \in \Theta^{*}$ and $M \subseteq \Theta^{\infty}$ is directed. Then $\sqcup M$ exists because $\left(\Theta^{\infty}, ㄷ\right)$ is a cpo (Theorem 4.2). We need to show the existence of $\tau \in M$ such that $\sigma \subseteq \tau$ if $\sigma \subseteq \sqcup M$. Suppose $\sigma \subseteq \sqcup M$. If $\sqcup M \in M$, then take $\tau=\sqcup M$. Otherwise, if $\sigma \subseteq \sqcup M$ and $\sqcup M \notin M$, then $M$ must be infinite by Lemma 4.1(ii); hence, $L M$ must be an infinite trace while $\sigma$ is its finite prefix, and thus $\sigma \neq \sqcup M$. Let $\gamma$ be an arbitrary member of $M$, then $\sigma, \gamma=\sqcup M$. It follows that $M \cup\{\sigma, \cup M\}$ is directed. Since $\sigma \neq \sqcup M$, we conclude $M \cup\{\sigma\}$ is directed. Thus, there must exist $\tau \in \mathrm{M}$ such that $\sigma, \gamma \sqsubseteq \tau$.

To show that elements of $\Theta^{\omega}$ are not the finite elements, take any $\sigma \in \Theta^{\omega}$ and the directed set $M=\downarrow^{\text {fin }} \sigma$. Then $\sigma=\sqcup M \notin M$ and for no $\tau \in M$ do we have $\sigma \equiv \tau$.

This concludes the proof.

Thus, finite trace prefices can be given the usual domain-theoretic interpretation of being a 'finite approximation' of a possibly infinite trace.

The following result provides us with an alternative construction for the domain of traces.

Lemma 4.9. Let $(\mathrm{D}$, 도) be a cpo. Then the following are equivalent:

(i) (D,, ) is algebraic,

(ii) $\mathrm{D}$ is isomorphic to the ideal-completion of a poset.

Proof. See e.g. [Law87].

Proposition 4.10. $\left(\Theta^{\infty}, \sqsubseteq\right)$ is isomorphic to the ideal completion of $\left(\Theta^{*}, \varsigma^{\mathrm{fin}}\right)$.

Proof. Direct from Lemma 4.9. 
Next, we summarize the remaining properties of the domain of traces.

Lemma 4.11. $\forall \sigma, \tau \in \Theta^{\infty}: \sigma \subseteq \tau \Leftrightarrow \downarrow^{\text {fin }} \sigma \subseteq \downarrow^{\text {fin }} \tau$.

Proof.

$\Rightarrow$ Follows from transitivity of $ᄃ$.

$\Leftrightarrow$ Direct from algebraicity of traces (Theorem 4.8 ).

Finally, the domain of traces can be shown to specialise to the domain of strings.

Proposition 4.12. Let $\mathrm{C}=(\mathrm{A}, \mathrm{l})$ be a concurrent alphabet such that $\mathrm{l}=\varnothing$. Then: $\left(\Theta^{\infty}, 5\right)$ is isomorphic to $\left(\mathrm{A}^{\infty}, \leq\right)$.

Proof. Easy; observe that if $\imath=\varnothing$ then traces are singleton sets.

\section{The Metric Space of Traces}

There exists a natural metric for traces defined as follows. Let $\sigma, \tau \in \Theta^{\infty}$ and put:

$$
\mathrm{d}_{\mathrm{tr}}(\sigma, \tau)=2^{-\inf \{\operatorname{len}(\gamma) \mid \gamma \in \sigma \Delta \tau\}}
$$

where len $(\gamma)$ denotes the length of some string $x \in \gamma, \inf \varnothing=+\infty$, and $\sigma \Delta \tau$ is the symmetric difference of the sets of finite prefices below $\sigma$ and $\tau$ respectively. Formally:

$$
\sigma \Delta \tau=\left(\downarrow^{\mathrm{fin}} \sigma \downarrow \downarrow\right) \cup\left(\downarrow^{\mathrm{fin}} \tau \downarrow \downarrow \sigma\right)
$$

Note that the value of $\inf \{\operatorname{len}(\gamma) \mid \gamma \in \sigma \Delta \tau\}$ is a measure of the 'depth' of approximation necessary to distinguish between traces $\sigma$ and $\tau$.

It can be shown $\left[\mathrm{Kwi}\right.$ wom] that $\mathrm{d}_{\mathrm{tr}}$ is an ultrametric and that the following holds.

Proposition 4.13. ([Kwi89m])

(i) $\left(\Theta^{\infty}, \mathrm{d}_{\mathrm{tr}}\right)$ is a complete metric space.

(iii) If $\mathrm{A}$ is finite then the $\mathrm{d}_{\mathrm{tr}}$-topology in $\Theta^{\infty}$ is the Lawson topology.

Also, the metric $d_{t r}$ specializes to the metric $d_{s t r}$ of $[B \circ N 80]$ in case of $\imath=\varnothing$. Thus, infinite traces have two characterizations: as metric limits of Cauchy sequences of finite traces, and as order-theoretic limits of directed sets of finite traces.

It is an interesting question to investigate the continuity properties of the concatenation of finite and traces. Unfortunately, unlike in the case of strings, concatenation is not uniformly continuous on $\Theta^{*}$ wrt the metric $d_{t r}$. To demonstrate this fact, we need to show that:

$$
\exists M \forall N \exists \sigma, \tau, \sigma^{\prime}, \tau^{\prime}:\left[d_{t r}\left(<\sigma, \tau,\left\langle\sigma^{\prime}, \tau^{\prime}\right\rangle\right)<2^{-N} \text { and } d_{t r}\left(\sigma \tau, \sigma^{\prime} \tau^{\prime}\right) \geq 2^{-M}\right] \text {. }
$$

Let $A=\{a, b, c)$ with a $\imath b$, and take $\sigma=\left[a^{n}\right], \sigma^{*}=\left[a^{n} c\right], \tau=\tau^{*}=[b]$. Then:

$$
\mathrm{d}_{\mathrm{tr}}\left(\sigma \tau, \sigma^{\prime} \tau^{\prime}\right)=\mathrm{d}_{\mathrm{tr}}\left(\left[\mathrm{a}^{\mathrm{n}} \mathrm{b}\right],\left[\mathrm{a}^{\mathrm{n}} \mathrm{cb}\right]\right)=2^{-1}
$$

because $[b]$ is the shortest prefix in $\left[a^{n} b\right] \Delta\left[a^{n} c b\right]=\left\{[b],\left[a^{n} c b\right],\left[a^{n} c b\right]\right\}$, and yet: 


$$
\begin{aligned}
& d_{t r}(<\sigma, \tau\rangle,\left\langle\sigma^{\prime}, \tau^{\prime}>\right)=\max \left\{d_{t r}\left(\sigma, \sigma^{\prime}\right), d_{t r}\left(\tau, \tau^{\prime}\right)\right\} \\
& =\max \left(d_{t r}\left(\left[a^{n}\right],\left[a^{n} c\right]\right), d_{t r}([b],[b])\right\}=\max \left\{2^{-(n+1)}, 0\right\}=2^{-(n+1)}
\end{aligned}
$$

because $\left[\mathrm{a}^{\mathrm{n}} \mathrm{c}\right]$ is the shortest prefix in $\left[\mathrm{a}^{\mathrm{n}}\right] \Delta\left[\mathrm{a}^{\mathrm{n}} \mathrm{c}\right]$. Thus, unlike in the case of strings, the concatenation over $\Theta^{*}$ does not have a canonical extension to $\Theta^{\infty}$.

\section{SEQUENTIAL COMPOSITION OF TRACES}

We now investigate the monoid properties of $\Theta^{\infty}$. We believe that concatenation in $\Theta^{\infty}$ should be a suitable generalization of the concatenation in $\mathrm{A}^{\infty}$, as the latter corresponds to our intuitive understanding of sequential composition of two (sequential) executions $x$ and $y$ : if the behaviour represented by $x$ does not terminate, then the behaviour represented by $y$ should never proceed. What is then the right intuition for defining sequential composition of two non-sequential executions represented by traces? Ideally, causal independency should be taken into account. Suppose . denotes the concatenation operation, then for $\mathrm{a}$ and $\mathrm{b}$ independent it would be against reasonable intuition to define $\left[\mathrm{a}^{0}\right] .[\mathrm{b}]$ as $\left[\mathrm{a}^{{ }^{0}}\right]$ because the occurrence of a can in no way affect $b$; thus, the occurrence of a should not prevent $b$ from proceeding. Observe that traces $\left[\mathrm{a}^{\omega}\right]$ and $[\mathrm{b}]$ are consistent, i.e. have a common bound, e.g. $\left[\mathrm{ba}{ }^{0}\right]$. On the other hand, if $a$ and $b$ are dependent, $\left[a^{\omega}\right] .[b]$ should be $\left[a^{\omega}\right]$. Note that in this case the traces $\left[a^{\omega}\right]$ and $[b]$ are inconsistent.

However, if we accept the above intuition regarding respecting causality, we will lose associativity.

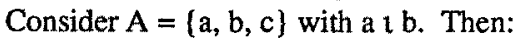

$$
\begin{aligned}
& {\left[a^{\infty}\right] \cdot([c] \cdot[b])=\left[a^{\infty}\right] \cdot([c b])=\left[a^{0}\right] \neq} \\
& \left(\left[a^{6}\right] \cdot[c]\right) \cdot[b]=\left[a^{0}\right] \cdot[b]=\left[b a^{\infty}\right]
\end{aligned}
$$

and $\left[\mathrm{a}^{\omega}\right] \neq\left[\mathrm{ba}^{\infty}\right]$. Moreover, continuity wrt the metric $\mathrm{d}_{\mathrm{tr}}$ fails for very similar reasons. We have:

$$
\lim \left(\left[a^{n} c\right] \cdot[b]\right)=\lim \left(\left[a^{n} c b\right]\right)=\left[a^{\infty}\right] \neq\left(\lim \left[a^{n} c\right]\right) \cdot(\lim [b])=\left[a^{\infty}\right] \cdot[b]=\left[b a^{\infty}\right]
$$

Scott continuity will also fail because the concatenation of finite traces is not even monotone. It remains to be seen if techniques similar to those used in Section 2 to show that concatenation of strings is Scottcontinuous can be applied in this case.

\section{Defining Sequential Composition}

We now introduce an extension of the concatenation of finite traces to $\Theta^{\infty}$ which agrees with the above intuition. We also show that $\left(\Theta^{\infty}, .,[\varepsilon]\right)$ is a pseudo-monoid, in the sense that. is total and associativity holds on a subset. Although non-associativity may be viewed as undesirable, our definition allows us to generalize Levi's lemma [Maz84, CoP85] to possibly infinite traces.

\section{Definition 5.1.}

(i) We say traces $\sigma, \tau$ are independent iff $\operatorname{Act}(\sigma) \times \operatorname{Act}(\tau) \subseteq 1$.

(ii) A trace $\tau \in \Theta_{l}^{\infty}$ is tail-independent with the trace $\sigma \in \Theta^{\infty}$ iff:

$\exists \sigma^{\prime} \check{c}^{\text {fin }} \sigma, \forall \sigma^{\prime \prime} \in \Theta^{*}: \sigma^{\prime} \sigma^{\prime \prime} \subseteq \sigma \Rightarrow \sigma^{\prime \prime}, \tau$ are independent.

(iii) A trace $\tau \in \Theta^{\infty}$ is tail-independent with the trace $\sigma \in \Theta^{\infty}$ after $\sigma^{\prime}$ iff 


$$
\begin{aligned}
& \sigma^{\prime} \sqsubseteq^{\text {fin }} \sigma \text { and } \\
& \forall \sigma^{\prime \prime} \in \Theta^{*}: \sigma^{\prime} \sigma^{\prime \prime} \subseteq \sigma \Rightarrow \sigma^{\prime \prime}, \tau \text { are independent. }
\end{aligned}
$$

The following may be shown.

\section{Proposition 5.2.}

(i) For every $\sigma \in \Theta^{\infty}, \sigma$ is tail-independent with [₹].

(ii) For all $\sigma, \tau \in \Theta^{\infty}$, if $\sigma$, $\tau$ independent then $\sigma, \tau$ tail-independent.

(iii) For all $\sigma, \tau \in \Theta^{*}$ :

if $\sigma, \tau$ tail-independent after $\sigma^{\prime}$, then $\sigma^{\prime} \tau$ and $\sigma$ are consistent.

Define an auxiliary operator $\Lambda(\sigma, \tau)$ for $\sigma, \tau \in \Theta^{\infty}$ as follows.

$$
\Lambda(\sigma, \tau)=\left\{\sigma^{\prime} \tau^{\prime} \mid \sigma^{\prime} \underline{\leftrightarrows}^{\text {fin }} \sigma, \tau^{\prime} \underline{\check{c}}^{\text {fin }} \tau \text { and } \tau^{\prime} \text { tail-independent with } \sigma \text { after } \sigma^{\prime}\right\}
$$

Note that $\downarrow^{\text {fin }} \sigma \subseteq \Lambda(\sigma, \tau)$. Intuitively, we break the 'past history' of the execution $\sigma$ into finite segments

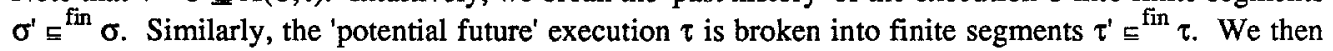
concatenate the finite past $\sigma^{\prime}$ with the finite future $\tau$ ' and choose only those segments $\sigma^{\prime} \tau^{\prime}$ which represent a viable continuation of the past history given by $\sigma$, in the sense of $\sigma^{\prime} \tau^{\prime}$ and $\sigma$ being consistent, i.e. having a common bound. Thus, $\Lambda(\sigma, \tau)$ represents all finite pieces of the new history. It is clear that for finite traces $\sigma$ and $\tau$ the whole of the trace $\sigma \tau$ becomes the new history; however, when traces $\sigma$ and $\tau$ are infinite, part of the future $\tau$ may be delayed indefinitely.

$\Lambda(\sigma, \tau)$ is the maximal directed subset of $\Sigma \mathrm{T}$ where $\sigma=\downarrow^{\mathrm{fin}} \sigma$ and $\mathrm{T}=\downarrow^{\mathrm{fin}} \tau$ that is consistent with $\sigma$. We can now define sequential composition in $\Theta^{\infty}$.

Definition 5.3. For $\sigma, \tau \in \Theta^{\infty}$.define:

$$
\sigma . \tau=\sqcup \Lambda(\sigma, \tau)
$$

\section{Proposition 5.4.}

(i) For all $\sigma, \tau \in \Theta^{\infty}, \sigma . \tau$ is well defined.

(ii) If $\sigma, \tau \in \Theta^{*}$, then $\Lambda(\sigma, \tau)=\downarrow^{\text {fin }_{[x y}}$, for some $\mathrm{x} \in \sigma, \mathrm{y} \in \tau$.

\section{Proof.}

(i) We need to show $\Lambda(\sigma, \tau)$ is a directed set. Note that $\Lambda(\sigma, \tau)$ is non-empty because $\downarrow^{\text {fin }} \sigma \subseteq \Lambda(\sigma, \tau)$

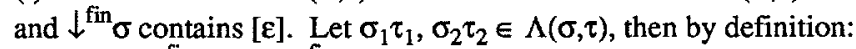

(a) $\sigma_{1} \stackrel{\text { fin }}{\subseteq} \sigma \wedge \tau_{1} \subseteq{ }^{\text {fin }} \tau \wedge \tau_{1}$ tail-independent with $\sigma$ after $\sigma_{1}$

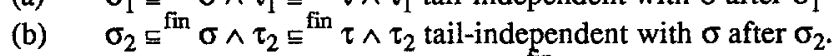

By Lemma 4.5 we have there exists $\sigma^{\prime} \check{5}^{\text {fin }} \sigma$ such that $\sigma^{\prime}=\sigma_{1} \nu \sigma_{2}$, and hence $\operatorname{Act}\left(\sigma^{\prime}\right)=$

$\operatorname{Act}\left(\sigma_{1}\right) \cup \operatorname{Act}\left(\sigma_{2}\right)$. Thus, by definition of tail-independency, $\tau_{1}$ must be tail-independent with $\sigma$ after $\sigma^{\prime}$ and $\tau_{2}$ tail-independent with $\sigma$ after $\sigma^{\prime}$. Using induction we can construct $\tau^{\prime} \check{c}^{\text {fin }} \tau$ such that $\tau^{\prime}=$ $\tau_{1} \sqcup \tau_{2}$. Then, $\operatorname{Act}\left(\tau^{\prime}\right)=\operatorname{Act}\left(\tau_{1}\right) \cup \operatorname{Act}\left(\tau_{2}\right)$. Let $\sigma^{\prime \prime} \in \Theta^{*}$ such that $\sigma^{\prime} \sigma^{\prime \prime} \unrhd^{\text {fin }} \sigma$. It is easy to see $\operatorname{Act}\left(\sigma^{\prime \prime}\right) \times \operatorname{Act}\left(\tau^{\prime}\right) \subseteq 1$; hence, $\sigma^{\prime \prime}$ and $\tau^{\prime}$ are independent. It follows $\tau^{\prime}$ is tail-independent with $\sigma$ after $\sigma^{\prime}$. Let $\sigma_{i} \sigma_{i}^{\prime \prime}=\sigma^{\prime}$ for $i \in\{1,2\}$. Then, since $\tau_{i}$, $\sigma_{i}$ " are independent, we have by Levi's lemma: $\sigma_{i} \tau_{i} \subseteq \sigma_{i} \tau_{i} \sigma_{i}^{\prime \prime}=\sigma_{i} \sigma_{i}^{\prime \prime} \tau_{i}=\sigma^{\prime} \tau_{i} \equiv \sigma^{\prime} \tau^{\prime}$.

We have thus constructed $\sigma^{\prime} \tau^{\prime} \in \Lambda(\sigma, \tau)$ such that $\sigma_{1} \tau_{1}, \sigma_{2} \tau_{2} \subseteq \sigma^{\prime} \tau^{\prime}$.

Since $\Theta^{\infty}$ is a cpo, $\sqcup \Lambda(\sigma, \tau)$ must exist.

(ii) Straightforward. 
Example 5.5. The following are examples of sequential composition in $\Theta^{\infty}$. Let $A=\{a, b, c\}$ with $a t b$, then:

$$
\begin{aligned}
& {[a] \cdot\left[b^{\omega}\right]=\sqcup\left(\left[b^{*}\right] \cup\left[a b^{*}\right]\right)=\left[a b^{\omega}\right],} \\
& {\left[a b^{\omega}\right] \cdot[a]=\sqcup\left(\{[\varepsilon]\} \cup\left[a b^{*}\right] \cup\left[a a b^{*}\right]\right)=\left[a a b^{\omega}\right],} \\
& \left.\left[a^{\omega}\right] \cdot\left[b^{\omega}\right]=\sqcup\left(\left[a^{*} b^{*}\right]\right)=\sqcup\left\{\left[\left(a^{*} b^{*}\right)^{\omega}\right]\right\}\right)=\left[(a b)^{\omega}\right]
\end{aligned}
$$

On the other hand:

$$
\begin{aligned}
& {[\mathrm{a}] \cdot\left[\mathrm{c}^{\omega}\right]=\sqcup\left(\{[\varepsilon]) \cup\left[\mathrm{ac}^{*}\right]\right)=\left[a c^{\omega}\right],} \\
& {\left[\mathrm{a}^{\omega}\right] \cdot[\mathrm{c}]=\sqcup\left(\left[\mathrm{a}^{*}\right]\right)=\left[\mathrm{a}^{\omega}\right]} \\
& {\left[\mathrm{a}^{\omega}\right] \cdot\left[\mathrm{c}^{\omega}\right]=\sqcup\left(\left[\mathrm{a}^{*}\right]\right)=\left[\mathrm{a}^{\omega}\right] .}
\end{aligned}
$$

Finally:

$$
\begin{aligned}
& {\left[\mathrm{ca}^{\omega}\right] \cdot[\mathrm{bc}]=\cup\left(\{[\varepsilon]\} \cup\left[\mathrm{ca}^{*}\right] \cup\{[\mathrm{cb}]\}\right)=\left[\mathrm{cba}^{\omega}\right],} \\
& {\left[\mathrm{ca}^{\omega}\right] \cdot[\mathrm{cb}]=\sqcup\left(\{[\varepsilon]\} \cup\left[\mathrm{ca}^{*}\right]\right)=\left[\mathrm{ca}^{\omega}\right] .}
\end{aligned}
$$

The following summarises the relationship of the sequential composition of possibly infinite strings and traces.

Proposition 5.6. If $\mathrm{l}=\varnothing$ then for any $\sigma, \gamma \in \Theta^{\infty}$ we have:

$$
\sigma . \gamma=[\mathrm{xy}]
$$

where $\mathrm{x} \in \sigma, \mathrm{y} \in \tau$.

Proof.

Straightforward.

The following important property may also be shown.

Lemma 5.7. (Shields) Suppose $\mathrm{x}, \mathrm{y} \in \Theta^{*}$, then for $\mathrm{z} \in \Theta^{\infty}$ : $(\mathrm{xy}) /(\mathrm{z} \sqcap(\mathrm{xy})) \geq \mathrm{x} /(\mathrm{z} \cap \mathrm{x})$.

Proof. By induction on the length of $y$ (see [Kwi89t]).

Proposition 5.8. Let $\sigma, \gamma \in \Theta^{\infty}$. Then

$$
\sigma \subseteq \gamma \Leftrightarrow \exists \beta \in \Theta^{\infty}: \sigma . \beta=\gamma \text {. }
$$

\section{Proof.}

$\Rightarrow$ Suppose $\sigma \subseteq \gamma$. We construct the least $\beta \in \Theta^{\infty}$ such that $\sigma . \beta=\gamma$. By Lemma 4.11 we have $\downarrow^{\text {fin }} \sigma \subseteq \downarrow^{\text {fin }} \gamma$. Define: where $M_{\tau}=\downarrow^{\text {fin }} \sigma \cap \downarrow^{\text {fin }} \tau$.

$$
M=\left\{\tau / \sigma^{*} \mid \tau \in\left(\downarrow^{\mathrm{fin}} \gamma\right) \backslash\left(\downarrow^{\mathrm{fin}} \sigma\right), \sigma^{\prime}=\sqcup \mathrm{M}_{\tau}\right\}
$$

(1) We prove that $M_{\tau}$ is directed and contains least upper bound of every pair of its elements. Observe that $M_{\tau}$ is non-empty because it contains $[\varepsilon]$. Let $\sigma_{1}, \sigma_{2} \in M_{\tau}$, then:

$$
\sigma_{1}, \sigma_{2} \subseteq \sigma \text { and } \sigma_{1}, \sigma_{2} \subseteq \tau
$$

By Lemma 4.5 it follows that $\sigma_{1} \sqcup \sigma_{2}$ exists and is bounded by $\sigma$ and $\tau$, and thus $\sigma_{1} \sqcup \sigma_{2} \in M_{\tau}$. Hence, $M_{\tau}$ is directed.

(2) Observe that $M$ is well-defined because $\sigma^{\prime}=\sqcup M_{\tau}$ always exists by (1) and $\sigma^{\prime} \subseteq \tau$. Also, $\sqcup M_{\tau}$ is finite because $\tau$ is finite.

(3) We show that $M$ is directed. Note that $M$ is non-empty because it contains $[\varepsilon]=\sqcup \varnothing$. Let $\beta^{\prime}, \beta^{\prime \prime} \in$ $M$, then $\beta^{\prime}=\tau^{\prime} / \sigma^{\prime}, \beta^{\prime \prime}=\tau^{\prime \prime} / \sigma^{\prime \prime}$. We need to show there exists $\beta^{\prime \prime \prime} \in M$ such that $\beta^{\prime}, \beta^{\prime \prime} \subseteq \beta^{\prime \prime \prime}$. Observe that $\tau^{\prime} \cup \tau^{\prime \prime}$ exists because $\tau^{\prime}, \tau^{\prime \prime}$ bounded by $\gamma$. Also, $\sigma^{\prime}=\sigma \sqcap \tau^{\prime}$ and $\sigma^{\prime \prime}=\sigma \sqcap \tau^{\prime \prime}$ (by definition). Let $\mathrm{y}=\left(\tau^{\prime} \cup \tau^{\prime \prime}\right) / \sigma$. By Lemma 5.7: 
$\left(\tau^{\prime} \cup \tau^{\prime \prime}\right) /\left(\sigma \sqcap\left(\tau^{\prime} \sqcup \tau^{\prime \prime}\right)\right)=\left(\tau^{\prime} y\right) /\left(\sigma \sqcap\left(\tau^{\prime} y\right)\right) \geq \tau^{\prime} /\left(\sigma \sqcap \tau^{\prime}\right)=\tau^{\prime} / \sigma^{\prime}$

By symmetry, $\left(\tau^{\prime} \sqcup \tau^{\prime \prime}\right) /\left(\sigma \sqcap\left(\tau^{\prime} \sqcup \tau^{\prime \prime}\right)\right) \supseteq \tau^{\prime \prime} /\left(\sigma \sqcap \tau^{\prime \prime}\right)=\tau^{\prime \prime} / \sigma^{\prime \prime}$. Note that $\left(\tau^{\prime} \sqcup \tau^{\prime \prime}\right) /\left(\sigma_{\sqcap}\left(\tau^{\prime} \sqcup \tau^{\prime \prime}\right)\right) \in \mathrm{M}$

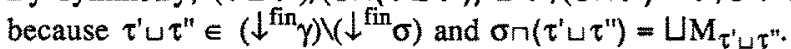

(4) Since $M$ directed by (3), define $\beta=L M$ (exists because $\Theta^{\infty}$ is a cpo by Theorem 4.2). It follows by construction that this is the least $\operatorname{such} \beta$.

(5) We show $\Lambda(\sigma, \beta) \subseteq \downarrow^{\text {fin }} \gamma$. Suppose $\sigma^{\prime} \beta^{\prime} \in \Lambda(\sigma, \beta)$, then by definition $\sigma^{\prime} \varsigma^{\text {fin }} \sigma, \beta^{\prime} \sqsubset^{\text {fin }} \beta$ and $\beta^{\prime}$ is tail-independent with $\sigma$ after $\sigma^{\prime}$. On the other hand, $\beta^{\prime}=\tau / \sigma^{\prime \prime}$ for some $\tau \in\left(\downarrow^{\text {fin }} \gamma\right) \backslash\left(\downarrow^{\text {fin }} \sigma\right), \sigma^{\prime \prime}=\sqcup M_{\tau}$ (by definition of $M$ ). Note that $\sigma^{\prime} \cup \sigma^{\prime \prime}$ exists, and that $\beta^{\prime}$ is tail-independent with $\sigma$ after $\sigma^{\prime} \cup \sigma^{\prime \prime}$.

Define $\kappa=\left(\sigma^{\prime} \sqcup \sigma^{\prime \prime}\right) \beta^{\prime}$ and observe that $\kappa \in \downarrow^{\text {fin }} \gamma$.

(6) We show $\downarrow^{\text {fin }} \gamma \subseteq \Lambda(\sigma, \beta)$. As $\downarrow^{\text {fin }} \sigma \subseteq \downarrow^{\text {fin }} \gamma$ it is sufficient to show that $\left.\left(\downarrow^{\text {fin }} \gamma\right) \backslash \downarrow^{\text {fin }} \sigma\right) \subseteq \Lambda(\sigma, \beta)$. Let $\tau \in\left(\downarrow^{\mathrm{fin}} \gamma\right) \backslash \downarrow^{\text {fin }} \sigma$ ) and define $\beta^{\prime}=\tau / \sigma^{\prime}$ where $\sigma^{\prime}=\sqcup \mathrm{M}_{\tau}$. Now $\beta^{\prime} \in \mathrm{M}$ (by definition of $M$ ), and thus $\beta^{\prime} \in \downarrow^{\text {fin }} \beta$. Finally observe that $\beta^{\prime}$ is tail-independent with $\sigma$ after $\sigma^{\prime}$.

$\Leftrightarrow$ Direct from definition of sequential composition and Lemma 4.11 .

Finally, we remark that associativity and metric continuity hold for pairs $<\sigma, \tau>$ such that $\sigma \in \Theta^{*}, \tau$ $\in \Theta^{\infty}$.

\section{Levi's Lemma for Infinite Traces}

The following property follows from the definition of sequential composition. It states that given any two consistent (i.e. bounded) traces, the corresponding continuations from their greatest lower bound commute. This property is referred to as the 'commutativity lemma'; it is a reformulation of the wellknown Levi's lemma for traces, which has been proved for the finite case in [Maz84, CoP85], and independently for the infinite case in [Gas90, Die91].

First, we extend the left cancellation operator on to $\Theta^{\infty}$ in the following way:

Definition 5.9. Let $\sigma, \gamma, \beta \in \Theta^{\infty}$ such that $\gamma \subseteq \sigma$. We say that $\beta$ is $\sigma$ after $\gamma$ (denoted $\sigma / \gamma$ ) iff $\beta$ is the least trace such that $\gamma \cdot \beta=\sigma$.

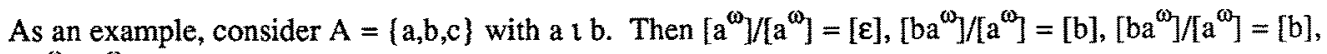
$\left[\mathrm{ca}^{0}\right] /\left[\mathrm{a}^{\mathrm{D}}\right]$ is undefined.

Proposition 5.10. (Commutativity lemma)

Let $\sigma, \tau, \beta \in \Theta^{\infty}$ such that $\sigma, \tau \sqsubseteq \beta$. Then:

$(\sigma / \gamma) \mathfrak{i}(\tau / \gamma)$ and $\sigma\llcorner\tau=\gamma .(\sigma / \gamma) .(\tau / \gamma)=\gamma .(\tau / \gamma) .(\sigma / \gamma)$

where $\gamma=\sigma \sqcap \tau$.

Sketch of proof. Observe that $\sigma u \tau$ exists by Lemma $4.5, \sigma \cap \tau$ exists by Proposition 4.6 , and both

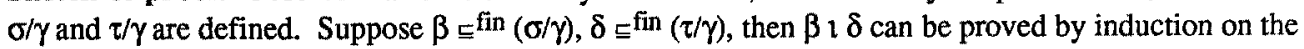
length of $\beta$; thus conclude $(\sigma / \gamma) \imath(\tau / \gamma)$. The equality $\sigma\lrcorner \tau=\gamma .(\sigma / \gamma) .(\tau / \gamma)=\gamma \cdot(\tau / \gamma) .(\sigma / \gamma)$ follows from the definition of sequential composition. 


\section{CONCLUSION}

The approach presented here has concentrated on a basic semantic framework that can be used to model concurrency in the non-interleaving sense, and its mathematical characterizations. We have investigated ways by which adding structural information in the form of the relation of causal independency over actions can influence the mathematical space of the infinitary behaviour of concurrent systems. This has been achieved by a suitable extension of trace theory, which has been shown to give rise to a Scott domain, a complete metric space and a pseudo-monoid. It has been demonstrated that there exists a natural approximation ordering on traces, which may be obtained by relaxing the corresponding approximation ordering on strings. Being less discriminating, trace prefix ordering allows to distinguish between action occurrences that represent either 'consistent' (i.e. co-existing, but perhaps remote) or 'inconsistent' (i.e. conflicting) pieces of some non-sequential 'history'.

The detailed investigation of existence of a Scott- and metric-continuous sequential composition, concurrent composition, and hiding operations is in progress. Further work will include techniques for representing process denotations in the domain of traces, deriving appropriate operations on process denotations and solving reflexive domain equations.

The mathematical structure of the space of finite and infinite traces is of considerable interest because it can be shown that using non-interleaving semantics has useful implications on fairness, see e.g. [Kwi90t, Kwi90c]. Maximality with respect to trace prefix ordering determines a certain notion of fairness [Kwi89e], and other notions of fairness, e.g. process fairness, also arise naturally [Kwi90p].

\section{ACKNOWLEDGEMENTS}

The author is particularly grateful to anonymous referees of an earlier version of this paper for drawing her attention to the failure of associativity. Mike Shields and Mike Smyth are also acknowledged for their help and many useful suggestions.

\section{REFERENCES}

[AaR88] Aalbersberg I.J., Rozenberg G., Theory of Traces, Theoretical Computer Science 60 (1988) 1-82.

[BoN80] Boasson L., Nivat M., Adherences of Languages, Journal of Computer and System Sciences 20 (1980) 285-309.

[CoP85] Cori R, Perrin D., Automates et commutations partielles, RAIRO Theoretical Informatics and Applications 19 (1985) 21-32.

[Die91] Diekert V., On the Concatenation of Infinite Traces, to appear in Proceedings, Symposium on Theoretical Aspects of Computer Science (STACS 91), Lecture Notes in Computer Science (Springer, 1991).

[Gas90] Gastin P., Infinite Traces, in I. Guessarian, ed., Semantics of Systems of Concurrent Processes, Lecture Notes in Computer Science 469 (Springer, 1990).

[GHK80] Gierz G., Hofmann K.H., Keimel K., Lawson J.D., Mislove M., Scott D., A Compendium of Continuous Lattices (Springer, 1980).

[Kwi90m] Kwiatkowska M.Z., A Metric for Traces, Information Processing Letters 35, 3 (1990). 
[Kwi89e] Kwiatkowska M.Z., Event Fairness and Non-Interleaving Concurrency, Formal Aspects of Computing 13 (1989).

[Kwi89t] Kwiatkowska M.Z., Fairness for Non-Interleaving Concurrency, PhD Thesis, University of Leicester (1989). Also available as Technical Report No. 22, University of Leicester, Department of Computing Studies (1989).

[Kwi90t] Kwiatkowska M.Z., On Topological Characterization of Behavioural Properties, to appear in: G.M. Reed \& A.W. Roscoe, eds., Topology in Computer Science (Oxford University Press, 1990).

[Kwi90c] Kwiatkowska M.Z., Causality and Faimess Properties, submitted.

[Kwi90p] Kwiatkowska M.Z., Defining Process Fairness for Non-Interleaving Concurrency, in K.V.Nori and C.E. Madhavan, eds., Foundations of Software Technology and Theoretical Computer Science, Lecture Notes in Computer Science 472 (Springer, 1990).

[Law87] Lawson J., The Versatile Continuous Order, in: M. Main et al, eds., Mathematical Foundations of Programming Language Semantics 1987, Lecture Notes in Computer Science 298 (Springer, 1988) 134-160.

[Maz77] Mazurkiewicz A., Concurrent Program Schemes and Their Interpretations, DAIMI Report PB - 78, Aarhus University (1977).

[Maz84] Mazurkiewicz A., Traces, Histories, Graphs: Instances of a Process Monoid, in: Chytil M.P., Koubek V., eds., Mathematical Foundations of Computer Science 1984, Lecture Notes in Computer Science 176 (Springer, 1984).

[Maz89] Mazurkiewicz A., Basic Notions of Trace Theory, in: J.W. de Bakker, W.-P. de Roever, G. Rozenberg, eds., Linear Time, Branching time and Partial Order in Logics and Models for Concurrency, Lecture Notes in Computer Science 354 (Springer, 1989) 285-263.

[Pnu86] Pnueli A., Applications of temporal logic to the specification and verification of reactive systems: a survey of current trends, in: de Bakker, de Roever, Rozenberg, eds., Current Trends in Concurrency, Lecture Notes in Computer Science 224 (Springer, 1986).

[Shi85] Shields M.W., Deterministic Asynchronous Automata, in: Formal Methods in Programming (North-Holland, 1985). 